\title{
Distributed Variance Regularized Multitask Learning
}

\author{
Michele Donini*, David Martinez-Rego ${ }^{\dagger}$, Martin Goodson ${ }^{\ddagger}$, John Shawe-Taylor ${ }^{\dagger}$, Massimiliano Pontil $^{\dagger \S}$ \\ *University of Padova, Padova, Italy \\ ${ }^{\dagger}$ University College London, Malet Place, London WC1E 6BT, UK \\ ${ }^{\ddagger}$ Skimlinks, London, UK \\ $\S$ Istituto Italiano di Tecnologia, Via Morego, Genoa, Italy
}

\begin{abstract}
Past research on Multitask Learning (MTL) has focused mainly on devising adequate regularizers and less on their scalability. In this paper, we present a method to scale up MTL methods which penalize the variance of the task weight vectors. The method builds upon the alternating direction method of multipliers to decouple the variance regularizer. It can be efficiently implemented by a distributed algorithm, in which the tasks are first independently solved and subsequently corrected to pool information from other tasks. We show that the method works well in practice and convergences in few distributed iterations. Furthermore, we empirically observe that the number of iterations is nearly independent of the number of tasks, yielding a computational gain of $O(T)$ over standard solvers. We also present experiments on a large URL classification dataset, which is challenging both in terms of volume of data points and dimensionality. Our results confirm that MTL can obtain superior performance over either learning a common model or independent task learning.
\end{abstract}

\section{INTRODUCTION}

Multitask Learning (MTL) is nowadays an established area of machine learning that has shown its benefits in many applications. MTL aims at simultaneously learning models for multiple related tasks by inducing some knowledge transfer between them. A typical MTL algorithm introduces a regularization term or prior that imposes an adequate shared bias between the learning tasks. This idea was inspired by research on transfer learning in psychology, which brought up the hypothesis that the abilities acquired while learning one task (e.g. to walk, to recognize cars, etc.) presumably apply when learning a similar task (to run, to recognize trucks, etc.) [Silver and Mercer, 1996], [Thrun, 1997] and was first coined inside the machine learning community in the 90's [Caruana, 1997], [Thrun, 1997]. The notion of what "task relatedness" means in practice is still not completely clear but theoretical studies support the intuition that training simultaneously from different related tasks is advantageous when compared to single task learning [Baxter et al., 2000] [Ben-David et al., 2003] [Maurer et al., 2006]. The benefits usually include more efficient use of small data sets in new related tasks and improved generalization bounds when learning simultaneously.

Recent successful studies on large datasets suggest that the use of models whose training process can be scaled up in terms of the size of the database is key to obtain good results. In large scale learning scenarios, the reduction of the estimation error that can be achieved by leveraging bigger datasets compensates the bias introduced by the use of simpler models such as linear classifiers [Bottou et al., 2008]. This trend has produced several algorithms that put the focus on scaling up the training of simple linear models such as support vector machines to PB of data, see [Shalev-Shwartz et al., 2014] and references therein. Despite this success, the MTL community has targeted the formulation of different regularizers that foster the correct information sharing between tasks in specific situations, overlooking their scalability. Nevertheless, MTL formulations apply very naturally to many large scale scenarios where we expect heterogeneous (although related) regimes to be present in the data.

Scalability of MTL can be challenged from two different angles. Recent applied studies on MTL [Birlutiu et al., 2013] [D'Avanzo et al. 2013] [Bai et al., 2012] [Huang et al., 2013], tackle scenarios such as preference learning, ranking, advertising targeting and content classification. Those studies mainly focus on the scalability when the number of examples increases. But scalability issues can also be encountered when the number of tasks grows. Think for example about learning: i) the preferences of a client on a per client basis, ii) the categorization of webpages on a per host basis or iii) the relevance of a query-document pair for a specific combination of user aspects like region, sex, age, etc. A common feature of these applications is that: (a) many tasks suffer a lack of data to learn from, and (b) the number of free parameters to learn grows with the number of tasks. The first issue has been one of the main motivations of multitask learning, namely to leverage data from similar tasks in order to improve accuracy. On the other hand, the second challenge could impose a limitation on the learning process if the number of tasks grows big since it means a larger amount of data to transfer on the network, stored and managed by the learning algorithms. Thus, if we combine MTL with parallel optimization, we would obtain a practical process that is expected to obtain higher accuracy.

\section{A. Our Contribution}

A key goal of this paper is to equip one of the most widespread MTL methods presented in [Evgeniou and Pontil, 2004] with a parallel optimization procedure. For this purpose, we employ the alternating direction method of multipliers (ADMM), see for example [Eckstein and Bertsekas, 1992], [Boyd, 2010] and references therein. We focus on MTL methods which involve the variance 
of the task weight vectors as the regularizer. We show that the optimization process can be efficiently implemented in a distributed setting, in that the different tasks can first be independently solved in parallel and subsequently corrected to pool information from other tasks. We report on numerical experiments, which indicate that the method works well and converges in few distributed iterations. We empirically observe that the number of iterations is nearly independent of the number of tasks, yielding a computational gain of $O(T)$ over standard solvers. We also present experiments on a large URL classification dataset, which is challenging both in terms of volume of data points, dimensionality and number of different tasks. Our results confirm that MTL can obtain superior performance over either learning a common model or independent task learning.

\section{B. Related Work}

The study of parallel MTL systems has been introduced in some previous works. In [Dinuzzo et al., 2011] the authors present a client-server MTL algorithm which maintains the privacy of the data between peers. Their method applies to the square loss function. In this paper we focus on the hinge loss, however our algorithm readily applies to other convex loss functions. It also preserves the privacy of data from different tasks since this does not need to leave client's side. In [Ahmed et al., 2014], the authors develop a parallel MTL strategy for hierarchies of tasks. This solution is based on Bayesian principles and presents some limitations when implemented on a MapReduce platform. In comparison, our approach takes a maximum margin approach that, although it does not include any hierarchy of tasks, maps naturally to any MapReduce environment and establishes the underpinnings to be extended to more complex situations. Very recently, in [Wang et al., 2015], the authors presented a distributed algorithm for group lasso multitask learning, addressing its statistical properties.

The paper is organized as follows. In Section 2 we briefly review the notation and the MTL method. We present our approach for making the optimization separable in Section 3. In Section 4, we describe in detail the main steps of the proposed algorithm. In Section 5 we present a stochastic gradient descent method to solve the inner SVM optimization problem and detail the convergence properties of this process. Experimental results with both artificial and real data are detailed in Section 6. Finally, In Section 7 we draw our conclusions and discuss perspectives for future work.

\section{BACKGROUND}

When we refer to multitask learning (MTL) we mean the following situation. We have $T$ learning tasks and we assume that all data from these tasks lie in the same space $\mathbb{R}^{d} \times Y$, where $Y=\{-1,1\}$ for binary classification and $Y=\mathbb{R}$ for regression. Associated with each task $t$ we have $m_{t}$ data points

$$
\left(\mathbf{x}_{1 t}, y_{1 t}\right),\left(\mathbf{x}_{2 t}, y_{2 t}\right), \ldots,\left(\mathbf{x}_{m_{t} t}, y_{m_{t} t}\right)
$$

sampled from a distribution $P_{t}$ on $X \times Y$. We assume that this distribution $P_{t}$ is different for each task but that different $P_{t}$ are related. The goal is to learn $T$ functions $h_{1}, h_{2}, \ldots, h_{T}$ such that the average error $\frac{1}{T} \sum_{i=1}^{T} \mathbb{E}_{(x, y) \sim P_{t}}\left[\ell\left(y, h_{t}(x)\right)\right]$ is low for a prescribed loss function $\ell$. Note that when $T=1$, this framework includes the single task learning problem as a specific case.

In [Evgeniou and Pontil, 2004] an intuitive formulation for relatedness between tasks inspired by a hierarchical bayesian perspective was presented. This formulation assumes that the hypothesis class of each individual task is formed by the set of linear predictors (classifiers) whose parameter vectors are related by the equations

$$
\mathbf{w}_{t}=\mathbf{v}_{t}+\mathbf{w}_{0}, \quad t=1, \ldots, T .
$$

The vector $\mathbf{v}_{t}$ models the bias for task $t$ and the vector $\mathbf{w}_{0}$ represents a common (mean) classifier between the tasks. Within this setting, the tasks are related when they are similar to each other, in the sense that the vectors $\mathbf{v}_{t}$ have small norms compared to the norm of the common vector $\mathbf{w}_{0}$. Note also that this setting may be useful in a transfer learning setting, in which we do not have data for a new task but we can still make predictions using the common average $\mathbf{w}_{0}$.

In the above setup, an optimal $\mathbf{w}_{0}$ and a set of optimal $\mathbf{v}_{t}$ for each task is found by solving the following optimization problem which is an extension of linear SVMs for a single task (which corresponds to the case $T=1$ ), namely

$$
\min _{\mathbf{w}_{0}, \mathbf{v}_{t}}\left\{\sum_{t=1}^{T} f_{t}\left(\mathbf{w}_{0}+\mathbf{v}_{t}\right)+\frac{\lambda_{1}}{T} \sum_{t=1}^{T}\left\|\mathbf{v}_{t}\right\|_{2}^{2}+\lambda_{2}\left\|\mathbf{w}_{0}\right\|_{2}^{2}\right\}
$$

where $f_{t}(\cdot)=\sum_{i=1}^{m_{t}} \ell\left(y_{i t},\left\langle\cdot, \mathbf{x}_{i t}\right\rangle\right)$, the empirical error for the task $t$.

Define the variance of the vectors $\mathbf{w}_{1}, \ldots, \mathbf{w}_{T}$ as

$$
\operatorname{Var}\left(\mathbf{w}_{1}, \ldots, \mathbf{w}_{T}\right)=\frac{1}{T} \sum_{t=1}^{T}\left\|\mathbf{w}_{t}-\frac{1}{T} \sum_{s=1}^{T} \mathbf{w}_{s}\right\|_{2}^{2} .
$$

It can be shown [Evgeniou and Pontil, 2004, Lemma 2.2] that problem (3) is equivalent to the problem

$\min _{\mathbf{w}_{t}}\left\{\sum_{t=1}^{T}\left(f_{t}\left(\mathbf{w}_{t}\right)+\rho_{1}\left\|\mathbf{w}_{t}\right\|_{2}^{2}\right)+\rho_{2} T \operatorname{Var}\left(\mathbf{w}_{1}, \ldots, \mathbf{w}_{T}\right)\right\}$

where the hyperparameters are linked by the equations

$$
\rho_{1}=\frac{1}{T} \frac{\lambda_{1} \lambda_{2}}{\lambda_{1}+\lambda_{2}}, \quad \rho_{2}=\frac{1}{T} \frac{\lambda_{1}^{2}}{\lambda_{1}+\lambda_{2}} .
$$

This connection makes it apparent that the regularization term encourages a small magnitude of the vectors $\mathbf{w}_{t}$ (large margin of each SVM) while simultaneously controlling their variance.

\section{Distributed MTL VIA ADMM}

The utility of problem (4) is that its objective function - unlike that in problem (3) - is almost separable across the different tasks. Namely, the only term in the objective function in (4) that prevents decoupling is the last summand, 
which makes the gradients for different tasks dependent. If we could remove this dependency, both the data and weight vectors for different tasks could be maintained in different nodes of a computer cluster without the need of centralizing any information and so making the method scalable. For this purpose we use an optimization strategy based on the alternating direction method of multipliers (ADMM), see for example [Boyd, 2010], [Eckstein and Bertsekas, 1992] and references therein. This method solve the general convex optimization problem

$$
\begin{array}{ll}
\underset{\mathbf{w}, \mathbf{z}}{\operatorname{minimize}} & f(\mathbf{w})+g(\mathbf{z}) \\
\text { subject to } & M \mathbf{w}=\mathbf{z}
\end{array}
$$

where $f$ and $g$ are two convex functions. Different algorithms have been proposed to tackle this optimization with different convergence characteristics, see, for example, [Boyd, 2010], [Mota et al., 2011], [He et al, 2012], [Hong, 2013]. In this paper we employ the ADMM algorithm outlined in [Eckstein and Bertsekas, 1992]. Although potentially faster algorithms exists, such as [Goldstein et al., 2014], their convergence analysis require stronger assumptions which are not meet in our problem.

We define the augmented Langrangian function $L_{\eta}$ at $\mathbf{w}, \mathbf{z}, \mathbf{y}$ as

$$
\begin{aligned}
L_{\eta}(\mathbf{w}, \mathbf{z}, \mathbf{y})=f(\mathbf{w})+g(\mathbf{z})+ & \langle\mathbf{y}, M \mathbf{w}-\mathbf{z}\rangle \\
& +\frac{\eta}{2}\|M \mathbf{w}+\mathbf{z}\|_{2}^{2}
\end{aligned}
$$

where $\eta$ is a positive parameter. Each ADMM step requires the following computations

$$
\begin{aligned}
\mathbf{w}^{k} & =\underset{\mathbf{x}}{\operatorname{argmin}} L_{\eta}\left(\mathbf{w}, \mathbf{z}^{k-1}, \mathbf{y}^{k-1}\right) \\
\mathbf{z}^{k} & =\underset{\mathbf{z}}{\operatorname{argmin}} L_{\eta}\left(\mathbf{w}^{k}, \mathbf{z}, \mathbf{y}^{k-1}\right) \\
\mathbf{y}^{k} & =\mathbf{y}^{k-1}+\eta\left(M \mathbf{w}^{k}-\mathbf{z}^{k}\right)
\end{aligned}
$$

where $k$ is a positive integer and $\mathbf{z}^{0}, \mathbf{y}^{0}$ are some starting points. We call each round of (6) an ADMM iteration. This process is repeated until convergence.

A convenient identification between problem (4) and the ADMM objective function (5) shows that the multitask objective can be efficiently optimized through this strategy. Namely, problem (4) is of the form (5) for the choice $M=I$ and

$$
\begin{aligned}
f(\mathbf{w}) & =\sum_{t=1}^{T} f_{t}\left(\mathbf{w}_{t}\right)+\frac{\rho_{1}}{2} \sum_{t=1}^{T}\left\|\mathbf{w}_{t}\right\|_{2}^{2} \\
g(\mathbf{z}) & =\rho_{2} T \operatorname{Var}\left(\mathbf{z}_{1}, \ldots, \mathbf{z}_{T}\right)+\frac{\rho_{1}}{2}\|\mathbf{z}\|_{2}^{2}
\end{aligned}
$$

where we set $\mathbf{w}$ to be the concatenation of the weight vectors $\mathbf{w}_{t}$ for all the tasks, and we force $\mathbf{z}=\mathbf{w}$ in order to make $f(\mathbf{w})+g(\mathbf{z})$ equal to the original objective in the feasible region.

The augmented Lagrangian for this specific case is

$$
\begin{aligned}
& L_{\eta}(\mathbf{w}, \mathbf{z}, \mathbf{y})=\sum_{t=1}^{T} f_{t}\left(\mathbf{w}_{t}\right)+\frac{\rho_{1}}{2} \sum_{t=1}^{T}\left(\left\|\mathbf{w}_{t}\right\|_{2}^{2}+\left\|\mathbf{z}_{t}\right\|_{2}^{2}\right) \\
& +\rho_{2} T \operatorname{Var}\left(\mathbf{z}_{1}, \ldots, \mathbf{z}_{T}\right)+\langle\mathbf{y}, \mathbf{w}-\mathbf{z}\rangle+\frac{\eta}{2}\|\mathbf{w}-\mathbf{z}\|_{2}^{2} .
\end{aligned}
$$

Using this expression, the first three updating equations in the ADMM optimization strategy (6) become

$$
\begin{gathered}
\mathbf{w}^{k}=\underset{\mathbf{w}}{\operatorname{argmin}}\left\{\sum_{t=1}^{T} f_{t}\left(\mathbf{w}_{t}\right)+\sum_{t=1}^{T} \frac{\rho_{1}}{2}\left\|\mathbf{w}_{t}\right\|_{2}^{2}\right. \\
\left.\quad+\left\langle\mathbf{y}_{t}^{k-1}, \mathbf{w}_{t}\right\rangle+\frac{\eta}{2}\left\|\mathbf{w}_{t}-\mathbf{z}_{t}^{k-1}\right\|_{2}^{2}\right\} \\
\mathbf{z}^{k}=\underset{\mathbf{z}}{\operatorname{argmin}}\left\{\rho_{2} T \operatorname{Var}\left(\mathbf{z}_{1}, \ldots, \mathbf{z}_{T}\right)+\frac{\rho_{1}}{2}\|\mathbf{z}\|_{2}^{2}\right. \\
\left.\quad-\left\langle\mathbf{y}^{k-1}, \mathbf{z}\right\rangle+\frac{\eta}{2}\left\|\mathbf{w}^{k-1}-\mathbf{z}\right\|_{2}^{2}\right\} \\
\mathbf{y}^{k}=\mathbf{y}^{k-1}+\eta\left(\mathbf{w}^{k}-\mathbf{z}^{k}\right) .
\end{gathered}
$$

We are left to analyze how to solve the first two optimization steps to obtain $\mathbf{w}^{k}$ and $\mathbf{z}^{k}$. It is noticeable that in the first of these steps the optimization over the tasks is completely decoupled, that is the component vectors $\mathbf{w}_{t}^{k}$ can be computed independently of each other - we discuss how to do this in Section V. Thus, the update of each task's weight vector can be run in parallel with no communication involved once data is distributed by tasks. As we shall see in Section IV-B the second optimization step, in which the only information sharing between the tasks occurs, can also be carried out with minimal communication just by averaging the vectors for the different tasks, hence leading to an scalable strategy.

\section{Algorithm}

In the previous section we showed how the MTL problem in equation (3) or (4) can be solved by the iterative scheme (6). In this section, we analyse the minimization problems for $\mathbf{w}^{k}$ and $\mathbf{z}^{k}$, noting that both can be computed in a distributed fashion.

\section{A. Optimization of each individual task}

The update formula for the weights $w$ in (7) can be implemented with different methods. The optimization of the weights completely decouple across the tasks $w_{t}$ and each can be compute by solving the problem

$$
\min _{\mathbf{w}_{t}}\left\{f_{t}\left(\mathbf{w}_{t}\right)+\frac{\rho_{1}+\eta}{2}\left\|\mathbf{w}_{t}\right\|_{2}^{2}+\left\langle\mathbf{y}_{t}-\eta \mathbf{z}_{t}, \mathbf{w}_{t}\right\rangle\right\} .
$$

One natural approach is to use (sub)gradient descent, possibly in a stochastic setting when the number of datapoints of a task is large. In this paper we consider the case that $\ell$ is the hinge loss, namely $\ell\left(y, y^{\prime}\right)=h\left(y y^{\prime}\right)$, where $h(\cdot)=\max (0,1-\cdot)$. In Section $\mathrm{V}$ we detail a stochastic gradient descent method to solve problem.

\section{B. Optimization of the auxiliary variables}

The update step for the auxiliary variable $\mathbf{z}^{k}$ is more advantageous computationally since we can work out a closed formula. The objective function is given by

$\rho_{2} \sum_{t=1}^{T}\left\|\mathbf{z}_{t}-\frac{1}{T} \sum_{s=1}^{T} \mathbf{z}_{s}\right\|_{2}^{2}+\frac{\rho_{1}}{2}\|\mathbf{z}\|_{2}^{2}+\left\langle\mathbf{y}^{k}, \mathbf{w}^{k}-\mathbf{z}\right\rangle+\frac{\eta}{2}\left\|\mathbf{w}^{k}-\mathbf{z}\right\|_{2}^{2}$ 
for fixed $\mathbf{w}^{k}$ and $\mathbf{y}^{k}$. Removing constant terms that do not depend on $\mathbf{z}$, this can further be rewritten as

$F(\mathbf{z})=\left\langle\mathbf{z},\left(\rho_{2} D^{T} D+\frac{\rho_{1}}{2} I\right) \mathbf{z}\right\rangle-\left\langle\mathbf{y}^{k}, \mathbf{z}\right\rangle+\frac{\eta}{2}\left(\|\mathbf{z}\|_{2}^{2}-2\left\langle\mathbf{w}^{k}, \mathbf{z}\right\rangle\right)$

where matrix $D \in \mathbb{R}^{T d \times T d}$ is given in block form as

$$
D=\left[\begin{array}{ccccc}
D_{M} & D_{0} & D_{0} & \cdots & D_{0} \\
D_{0} & D_{M} & D_{0} & \cdots & D_{0} \\
D_{0} & D_{0} & D_{M} & \cdots & D_{0} \\
\vdots & \vdots & \vdots & \vdots & \vdots \\
D_{0} & D_{0} & D_{0} & \cdots & D_{M}
\end{array}\right]
$$

being $d$ the number dimensions of a block and $T$ the number of blocks. Finally, $D_{M}=\frac{T-1}{T} I_{d \times d}$ and $D_{0}=-\frac{1}{T} I_{d \times d}$, where $I_{d \times d}$ is the $d \times d$ identity matrix.

If we take the derivative of the above objective function we can see that the optimal solution can be found by solving the system of linear equations

$$
\left(2 \rho_{2} D^{T} D+\left(\rho_{1}+\eta\right) I\right) \mathbf{z}^{k}=\left(\mathbf{y}^{k}+\eta \mathbf{w}^{k}\right) .
$$

The following lemma shows that the inverse $E=$ $\left(2 \rho_{2} D^{T} D+\left(\rho_{1}+\eta\right) I\right)^{-1}$ has a convenient closed analytical formula.

Lemma 1. Let $E=\left(2 \rho_{2} D^{T} D+\left(\rho_{1}+\eta\right) I\right)^{-1}$, where matrix $D \in \mathbb{R}^{T d \times T d}$ is defined equation (9). The matrix $E$ has the following structure

$$
E=\left[\begin{array}{ccccc}
E_{M} & E_{0} & E_{0} & \cdots & E_{0} \\
E_{0} & E_{M} & E_{0} & \cdots & E_{0} \\
E_{0} & E_{0} & E_{M} & \cdots & E_{0} \\
\cdots & \cdots & \cdots & \cdots & \cdots \\
E_{0} & E_{0} & E_{0} & \cdots & E_{M}
\end{array}\right] \in \mathbb{R}^{T d \times T d}
$$

where $E_{M}$ and $E_{0}$ are:

$$
\begin{aligned}
E_{M} & =\frac{1}{T}\left(\frac{1}{\eta+\rho_{2}}+\frac{T-1}{\eta+2 \rho_{1}+\rho_{2}}\right) I_{d \times d}, \\
E_{0} & =\frac{2 \rho_{1}}{T\left(\eta+\rho_{2}\right)\left(\eta+2 \rho_{1}+\rho_{2}\right)} I_{d \times d} .
\end{aligned}
$$

Proof. We have to prove that $\left(2 \rho_{2} D^{T} D+\left(\rho_{1}+\eta\right) I\right) E=$ $E\left(2 \rho_{2} D^{T} D+\left(\rho_{1}+\eta\right) I\right)=I \in \mathbb{R}^{T d \times T d}$. The first observation of the proof is the particular structure of the matrix $D$ that is an idempotent symmetric matrix and then $D^{T} D=D^{2}=D$. So, the matrix $2 \rho_{2} D^{T} D+\left(\rho_{1}+\eta\right) I$ is also equal to $G=$ $2 \rho_{2} D+\left(\rho_{1}+\eta\right) I$ and has the following structure in $\mathbb{R}^{T d \times T d}$

$$
\left[\begin{array}{cccc}
G_{M} & G_{0} & \cdots & G_{0} \\
G_{0} & G_{M} & \cdots & G_{0} \\
\cdots & \cdots & \cdots & \cdots \\
G_{0} & G_{0} & \cdots & G_{M}
\end{array}\right]
$$

where $G_{0}=2 \rho_{2} D_{0}, G_{M}=2 \rho_{2} D_{M}+\left(\rho_{1}+\eta\right) I_{d \times d}$, where $I_{d \times d}$ is the identity matrix in $d$ dimensions. Then, we can evaluate the block product between $E$ and $G$. This product generates a block matrix where each block on the diagonal is

$$
(T-1) E_{0} 2 \rho_{2} D_{0}+E_{M}\left(2 \rho_{2} D_{M}+\left(\rho_{1}+\eta\right) I_{d \times d}\right)=I_{d \times d},
$$

and the off-diagonal block are zero, since

$$
\begin{aligned}
(T-2) E_{0} 2 \rho_{2} D_{0}+E_{0}\left(2 \rho_{2} D_{M}+\right. & \left.\left(\rho_{1}+\eta\right) I_{d \times d}\right) \\
& +E_{M} 2 \rho_{2} D_{0}=0_{d} .
\end{aligned}
$$

This formula reveals that the optimization over $\mathbf{z}_{1}, \ldots, \mathbf{z}_{T}$ can also be run in parallel. First, we can reduce the vectors from all the tasks to a single vector $\mathbf{w}=E_{0} \sum_{t=1}^{T} \mathbf{w}_{t}$. Then, each vector can be updated in parallel by using again $E_{M}$ and $E_{0}$. Similarly to the optimization of $\mathbf{w}_{t}$, this operation can be readily done in a framework such as MapReduce to speed up computations with a very reduced need of broadcasting information.

\section{Convergence}

We comment on the convergence properties of our method. Our observations are a direct consequence of the general analysis in [Eckstein and Bertsekas, 1992]. Specifically, [Eckstein and Bertsekas, 1992, Theorem 8] applies to our problem, with (using their notation) $\rho_{k}=1$ for every $k \in \mathbb{N}$, their $\mathbf{p}$ equal to our $\mathbf{y}$ and their $\lambda$ equal our $\eta$. The theorem requires that the matrix $M$ is full rank, $f$ and $g$ are closed proper convex functions and the sum of the errors of the inner optimization problems is finite. All these hypothesis are meet in our case. In particular, the optimization over $\mathbf{z}$ is performed exactly as discussed in Section IV-B. The optimization over w are the parallel SVMs which we can solve to arbitrary precision using for example gradient descent. As we will see in our numerical experiments only few iterations are sufficient to reach a good suboptimal solution and gradient descent may be replaced by its stochastic version (discussed below) without affecting the good convergence of the algorithm.

\section{SGD OPTIMIZATION OF EACH INDIVIDUAL TASK}

When we have to solve problems with a large number of points, it is not computationally feasible to solve the optimization of each individual task with batch algorithms. In this section, we observe that it is possible to exploit a Stochastic Gradient Descent (SGD) strategy for our proposed method. Firstly, we show that the optimal solution of the optimization problem is contained inside a convex ball. From this results, we are able to satisfy the hypothesis of convergence of the SGD technique for strongly convex functions [Rakhlin et al., 2012], [Shalev-Shwartz et al., 2014]. Specifically, we prove the boundedness of the directions followed in the SGD optimization of $\mathbf{w}$, in each outer step $k \geq 0$ of the ADMM strategy. 
Algorithm 1 depicts the optimization of the weight vectors $\mathbf{w}_{t}$ using the SGD for strongly convex functions. The optimization for each task presented in Equation 8 is equivalent to the problem $\min _{\mathbf{w}_{t}} F_{t}\left(\mathbf{w}_{t}\right)$, where

$$
\begin{gathered}
F_{t}\left(\mathbf{w}_{t}\right)=L_{t}\left(\mathbf{w}_{t}\right)+\frac{\gamma_{t}}{2}\left\|\mathbf{w}_{t}\right\|_{2}^{2}, \\
L_{t}\left(\mathbf{w}_{t}\right)=\frac{1}{m_{t}}\left(\sum_{i=1}^{m_{t}} h\left(y_{i t}\left\langle\mathbf{w}_{t}, \mathbf{x}_{i t}\right\rangle\right)+\left\langle\mathbf{y}_{t}-\eta \mathbf{z}_{t}, \mathbf{w}_{t}\right\rangle\right)
\end{gathered}
$$

and $\gamma_{t}=\left(\rho_{1}+\eta\right) / m_{t}$. In order to be able to apply SGD we need to sample a direction $\mathbf{u}_{t}$ at each step $q$ such that $\mathbb{E}\left[\mathbf{u}_{t} \mid \mathbf{w}_{t}\right]$ is a subgradient of $F_{t}$ at $\mathbf{w}_{t}$. If at each step we sample randomly and with replacement a pattern $\mathbf{x}_{i t}$ from the training set, the following sequence of directions complies with the above restriction

$$
\mathbf{u}_{t}= \begin{cases}\gamma_{t} \mathbf{w}_{t}+\frac{1}{m}\left(\mathbf{y}_{t}-\eta \mathbf{z}_{t}\right) & \text { if } y_{i t}\left\langle\mathbf{w}_{t}, \mathbf{x}_{i t}\right\rangle \geq 1, \\ -y_{i t} \mathbf{x}_{i t}+\gamma_{t} \mathbf{w}_{t}+\frac{1}{m}\left(\mathbf{y}_{t}-\eta \mathbf{z}_{t}\right) & \text { otherwise. }\end{cases}
$$

In the following lemma, we show that the optimal solution of the original optimization problem resides inside a convex ball.

Lemma 2. If $\mathbf{w}^{*}$ is the optimal solution of problem (4) then $\left\|\mathbf{w}^{*}\right\|_{2} \leq \sqrt{\frac{T M}{2 \lambda_{1}}}$, where $M=\sum_{t=1}^{T} m_{t}$.

Proof. Let $\mu=\frac{T \lambda_{2}}{\lambda_{1}}$. We make the change of variables $\mathbf{w}=$ $\left(\sqrt{\mu} \mathbf{w}_{0}, \mathbf{v}_{\mathbf{1}}, \ldots, \mathbf{v}_{T}\right) \in \mathbb{R}^{(T+1) d}$ and introduce the map $\phi:$ $\mathbb{R}^{d} \times\{1, \ldots, T\} \mapsto \mathbb{R}^{(T+1) d}$, defined as

$$
\phi(\mathbf{x}, t)=\left(\frac{\mathbf{x}}{\sqrt{\mu}}, 0, \ldots, 0, \mathbf{x}, 0, \ldots, 0\right) .
$$

Following [Evgeniou and Pontil, 2004] we rewrite problem (3) as a standard SVM problem in the extended input space for the feature map (11), namely

$$
\min _{\mathbf{w}}\left\{C \sum_{i=1}^{M} h\left(y_{i}\left\langle\mathbf{w}, \phi\left(\mathbf{x}_{i}, t_{i}\right)\right\rangle\right)+\frac{1}{2}\|\mathbf{w}\|_{2}^{2}\right\}
$$

where $C=\frac{T}{2 \lambda_{1}}$ and $M=\sum_{t=1}^{T} m_{t}$. Since at the optimum the gap between the primal and dual objective vanishes, there exists $\boldsymbol{\alpha}^{*} \in[0, C]^{M}$ such that

$$
\frac{1}{2}\left\|\mathbf{w}^{*}\right\|_{2}^{2}+C \sum_{i=1}^{M} h\left(y_{i}\left\langle\mathbf{w}, \phi\left(\mathbf{x}_{i}, t_{i}\right)\right\rangle\right)=\sum_{i=1}^{M} \boldsymbol{\alpha}_{i}^{*}-\frac{1}{2}\left\|\mathbf{w}^{*}\right\|_{2}^{2} .
$$

Using the fact that $\sum_{i=1}^{M} h\left(y_{i}\left\langle\mathbf{w}, \phi\left(\mathbf{x}_{i}, t_{i}\right)\right\rangle\right) \geq 0$ we conclude that $\left\|\mathbf{w}^{*}\right\|_{2}^{2} \leq \sum_{i=1}^{M}{ }^{i=1} \boldsymbol{\alpha}_{i}^{*} \leq M C=\frac{T M}{2 \lambda_{1}}=\beta$.

This lemma thus proves that in Algorithm 1 it is safe to restrict the set of feasible solutions to the ball $\mathbb{B}:=\left\{\mathbf{x} \in \mathbb{R}^{d}:\|\mathbf{x}\|_{2}^{2} \leq \beta\right\}$ with $\beta=\max \left(\sqrt{T M /\left(2 \lambda_{1}\right)}, \sqrt{M /\left(2 \lambda_{1}\right)}\right)$ and it justifies the projection step at line 7 of the algorithm.
Algorithm 1 Optimization of each individual task

Input: Dataset $\left\{\left(\mathbf{x}_{i t}, y_{i t}\right), i=1, \ldots, m_{t}\right\}$ for task $t$, parameters $\rho_{1}, \gamma_{t}, \eta, \mathbf{z}_{t}, \mathbf{y}_{t}, T, M$

Output: Optimal weight vector $\mathbf{w}_{t}$

$$
\begin{aligned}
& \text { 1: } \mathbf{w}_{t}^{0} \leftarrow \mathbf{0} \\
& \text { 2: } \gamma_{t} \leftarrow \frac{\rho_{1}+\eta}{m_{t}} \\
& \text { 3: for } q \leftarrow 1 \text { to } n \text { do } \\
& \text { 4: } \quad \delta \leftarrow \frac{1}{q \gamma_{t}}
\end{aligned}
$$$$
\text { 5: } \quad \text { Choose a random pattern }\left(\mathbf{x}_{i t}, y_{i t}\right) \text { from the set }
$$$$
\text { 6: } \quad \mathbf{w}_{t}^{q+\frac{1}{2}} \leftarrow \mathbf{w}_{t}^{q}-\delta \mathbf{u}_{t}
$$$$
\text { 7: } \quad \mathbf{w}_{t}^{q+1} \leftarrow \min \left(1, \beta \frac{1}{\left\|\mathbf{w}_{t}^{q+\frac{1}{2}}\right\|_{2}}\right) \mathbf{w}_{t}^{q+\frac{1}{2}}
$$$$
\text { 8: return } \hat{\mathbf{w}}_{t}=\frac{2}{n} \sum_{q=\frac{n}{2}+1}^{n} \mathbf{w}_{t}^{q}
$$

Now, we analyze the convergence of Algorithm 1 and we base our proof on a recent result presented in [Rakhlin et al., 2012], [Shalev-Shwartz et al., 2014] which states (adapting their result to our notation) that the aforementioned strategy converges with the rate $\mathbb{E}\left[F_{t}\left(\hat{\mathbf{w}}_{t}\right)\right]-F_{t}\left(\mathbf{w}_{t}\right) \leq$ $\frac{\rho^{2}}{2 \gamma_{t} n}$ after $n$ iterations, provided when $F_{t}(\mathbf{w})$ is $\gamma_{t}$-strongly convex and $\mathbb{E}\left[\left\|\mathbf{u}_{t}\right\|^{2}\right] \leq \rho^{2}$ for some finite constant $\rho$. Clearly, the objective function $F_{t}$ is strongly convex with constant $\gamma_{t}=\frac{\rho_{1}+\eta}{m_{t}}$, see [Shalev-Shwartz et al., 2014, Lemma 13.5]. We are left to prove that the directions $\mathbf{u}_{t}$ followed in the SGD optimization of $\mathbf{w}$ in each outer step $k \geq 0$ of the ADMM strategy are bounded, which we state in the following theorem.

Theorem 1. If all the examples $\mathbf{x}_{i t}$ satisfy $\left\|\mathbf{x}_{i t}\right\|_{2}^{2} \leq R$ then for every iteration $k \in \mathbb{N}$ of the outer ADMM scheme and every inner iteration $q$, there exists $\rho>0$ such that the directions of the $S G D$ in Algorithm 1 are bounded in average, $\mathbb{E}\left[\left\|\mathbf{u}_{t}^{k}\right\|_{2}^{2}\right] \leq$ $\rho^{2}<+\infty$ for every $t=1, \ldots, T$.

Proof. At each outer step $k$, the direction for the task $t$ is:

$$
\mathbf{u}_{t}^{k}= \begin{cases}\gamma_{t} \mathbf{w}_{t}^{k}+\frac{1}{m}\left(\mathbf{y}_{t}^{k}-\eta \mathbf{z}_{t}^{k}\right) & \text { if } \ell_{i t}^{k}=0, \\ -y_{i t} \mathbf{x}_{i t}+\gamma_{t} \mathbf{w}_{t}^{k}+\frac{1}{m}\left(\mathbf{y}_{t}^{k}-\eta \mathbf{z}_{t}^{k}\right) & \text { if } \ell_{i t}^{k}>0\end{cases}
$$

where we use the shorthand $\ell_{i t}^{k}=y_{i t}\left\langle\mathbf{w}_{t}, \mathbf{x}_{i t}\right\rangle$.

We are interested in finding a bound for the quantity $\left\|\mathbf{u}_{t}^{k}\right\|_{2}^{2}$. From the definition we have that

$$
\left\|\mathbf{u}_{t}^{k}\right\|_{2}^{2} \leq\left\|y_{i t} \mathbf{x}_{i t}\right\|_{2}^{2}+\gamma_{t}^{2}\left\|\mathbf{w}_{t}^{k}\right\|_{2}^{2}+\frac{1}{m^{2}}\left(\left\|\mathbf{y}_{t}^{k}\right\|_{2}^{2}+\eta^{2}\left\|\mathbf{z}_{t}^{k}\right\|_{2}^{2}\right) \text {. }
$$

We have already shown that $\left\|\mathbf{w}_{t}^{k}\right\|_{2}^{2} \leq \beta$ and from the hypothesis we have that $\left\|y_{i t} \mathbf{x}_{i t}\right\|_{2}^{2} \leq R$. Now, we are interested in finding a finite bound for the quantity $\left\|\mathbf{y}_{t}^{k}\right\|_{2}^{2}+\eta^{2}\left\|\mathbf{z}_{t}^{k}\right\|_{2}^{2}$. From the initialization of the algorithm, we have that $\left\|\mathbf{y}_{t}^{1}\right\|_{2}^{2}+$ $\eta^{2}\left\|\mathbf{z}_{t}^{1}\right\|_{2}^{2}<+\infty$. In fact, $\left\|\mathbf{y}_{t}^{1}\right\|_{2}^{2}<+\infty$ and $\left\|\mathbf{z}_{t}^{1}\right\|_{2}^{2}<+\infty$. We can use the induction for the value $\left\|\mathbf{y}_{t}^{k}\right\|_{2}^{2}+\eta^{2}\left\|\mathbf{z}_{t}^{k}\right\|_{2}^{2}$ changing the step $k$. We can exploit the hypothesis that

$$
\left\|\mathbf{y}_{t}^{i}\right\|_{2}^{2}+\eta^{2}\left\|\mathbf{z}_{t}^{i}\right\|_{2}^{2}<+\infty, \quad \forall i<k
$$

and then $\left\|\mathbf{y}_{t}^{i}\right\|_{2}^{2}<+\infty$ and $\eta^{2}\left\|\mathbf{z}_{t}^{i}\right\|_{2}^{2}<+\infty, \quad \forall i<k$. 
By definition, the following inequalities hold

$$
\begin{aligned}
\left\|\mathbf{y}_{t}^{k}\right\|_{2}^{2} & \leq\left\|\mathbf{y}_{t}^{k-1}\right\|_{2}^{2}+\left\|\eta \mathbf{w}_{t}^{k-1}\right\|_{2}^{2}+\left\|\eta \mathbf{z}_{t}^{k-1}\right\|_{2}^{2} \\
& \leq\left\|\mathbf{y}_{t}^{k-1}\right\|_{2}^{2}+\eta^{2} \beta+\eta^{2}\left\|\mathbf{z}_{t}^{k-1}\right\|_{2}^{2} \\
& \leq\left\|\mathbf{y}_{t}^{1}\right\|_{2}^{2}+\sum_{i=2}^{k-1} \eta^{2}\left(\beta+\left\|\mathbf{z}_{t}^{i}\right\|_{2}^{2}\right)=: \Phi_{t}^{k} .
\end{aligned}
$$

By the induction hypothesis the value $\Phi_{t}^{k}<+\infty$. Also, we have that

$$
\left\|\mathbf{z}_{t}^{k}\right\|_{2}^{2} \leq\|E\|_{2}^{2}\left(\left\|\mathbf{y}_{t}^{k}\right\|_{2}^{2}+\eta^{2}\left\|\mathbf{w}_{t}^{k}\right\|_{2}^{2}\right)
$$

where $E=\left(2 \rho_{2} D^{T} D+\left(\rho_{1}+\eta\right) I\right)^{-1}$ is symmetric and then $\|E\|_{2}^{2}$ is its spectral radius $r_{E} \in \mathbb{R}^{+}$. Then, we can claim that

$$
\left\|\mathbf{z}_{t}^{k}\right\|_{2}^{2} \leq r_{E}\left(\left\|\mathbf{y}_{t}^{k}\right\|_{2}^{2}+\eta^{2} \beta\right) \leq r_{E}\left(\Phi_{t}^{k}+\eta^{2} \beta\right)=: \Psi_{t}^{k}<+\infty .
$$

Finally, the following bound holds:

$$
\left\|\mathbf{u}_{t}^{k}\right\|_{2}^{2} \leq R+\gamma_{t}^{2} \beta+\frac{1}{m^{2}}\left(\Phi_{t}^{k}+\eta^{2} \Psi_{t}^{k}\right):=\rho^{2}<+\infty .
$$

\section{EXPERIMENTAL RESULTS}

In this section present numerical experiments which highlight the computational efficiency of our algorithm, and report on the advantage offered by the MTL strategy in formula (3) in a challenging url classification dataset. Our implementation is available at https://github.com/torito1984/MTLADMM

\section{A. Artificial data}

In the first experiment we generated an artificial dataset which is captured by the model (2) and, in addition, introduces a set of irrelevant features.

The number of relevant and irrelevant features was set to 8 and 100, respectively. The dataset is made of 400 different binary classification tasks, 5 of which have 1000 patterns and the rest 16 patterns. To generate the data we first sample the components $w_{01}, \ldots, w_{0 d}$ of the mean vector $\mathbf{w}_{0}$ iid form a zero mean Gaussian with variance $\sigma=0.25$. Then, we randomly pick 5 of the relevant features and create a vector $\mathbf{w}_{t}$ for each task by adding a Gaussian perturbation with zero mean and standard deviation $\sigma_{i}=2 w_{0 i}$. Next for each task, we generate a balanced set of points on each side of the classification hyperplane. To generate a point we: (1) pick a sign $s$ with a $50 \%$ chance, (2) starting from the origin, move in a random direction $s \mathbf{d}$ where $\mathbf{d}$ is the normal vector of the classification hyperplane and $p \in N(5,0.1)$ (this generates a well behaved dataset with some random margin violations), (3) finally we choose a random direction in the subspace of dimension $d-1$ parallel to the classification hyperplane and make a jump with magnitude sampled from $N(0,20)$. Since we are generating patterns with a low margin covering many different directions in the parallel subspace, only the tasks with 1000 patterns have enough data to achieve high test accuracy by their own. For the rest of the tasks - which account for around $55 \%$ of the dataset - the number of points is insufficient to achieve a reasonable solution and they should benefit from

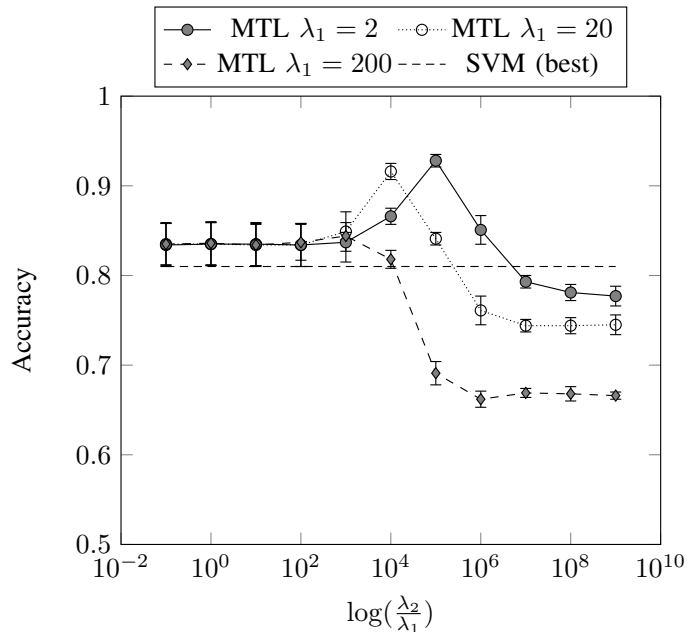

Fig. 1. Classification performance for the artificial classification dataset

an MTL approach. We will see that a similar situation arises in the real dataset we cover hereunder.

Returning to formula (3), it is instructive to observe that, for a fixed $\lambda_{1}>0$, if we let $\lambda_{2} \rightarrow \infty$, the optimization problem is equivalent to training separate linear SVMs on each task. In this situation and in high dimension, the method will overfit the dataset. On the other hand, for a fixed $\lambda_{2}>0$, if we let $\lambda_{1} \rightarrow \infty$, the optimization problem is equivalent to training a single linear SVMs on the full dataset, which may underfit the data. In practical situations, we expect the optimal hyperparameters to lay in between these two situations, and obtaining lower accuracy for both extremes.

1) Classification performance: Figure 1 depicts the average test accuracy for a 10-fold CV in the aforementioned dataset. Solid lines depict the accuracy of the proposed algorithm for different values of $\lambda_{1}$ and $\lambda_{2}$. As expected, the optimal hyperparameters lay in between both extremes for $\lambda_{2} / \lambda_{1}$ and overfitting arises for when $\lambda_{2} \gg \lambda_{1}$. The dotted line depicts the accuracy of a single SVM trained with a state of the art optimization algorithms for linear SVMs in [Hsieh et al., 2008]. We can observe that, with appropriate hyperparameters, MTL is superior to single task learning thanks to the information sharing.

2) Convergence: One question that may arise is how many ADMM iterations are needed to achieve a good result in practice. This would be the major bottleneck of the proposed method since ADMM iterations are sequential. We ran experiments with the same data generation strategy and varying the dimensionality. The number of relevant features was kept at $10 \%$ of the dimensionality.

First, in Figure 2, it can be observed that a higher dimension slows down the convergence to the optimum in the first iterations, an order of tens of iterations is enough to obtain a good accuracy. Second, we compared a MatLab implementation of the standard MTL called MALSAR ${ }^{1}$ in performance and

\footnotetext{
${ }^{1}$ MALSAR code: http://www.public.asu.edu/ jye02/Software/MALSAR
} 
computational complexity varying the number of different tasks in the set $\{20,40,60,80,100,200,400,800,1200\}$. We keep the sample sizes per task fixed and measure MALSAR running time. For our method, we are interested in finding the number of outer ADMM iterations needed to converge to the same accuracy of MALSAR with a tolerance of $10^{-4}$.

In Table I a comparison of the computational complexities is presented. We collected the CPU time required by the standard MTL implementation in order to find the optimal solution, varying the number of tasks. We compared these values with respect to the number of outer ADMM steps that our algorithm required to reach the same solution. For this purpose, we introduce the variable $\alpha=\frac{\lambda_{2}}{\lambda_{1}+\lambda_{2}}$. This variable imposes the quantity of shared information among the tasks. In fact, when $\alpha$ is equal to zero, we are training a single SVM for all the tasks, on the other hand, with $\alpha=1$ we are training a different SVM for each single task.

In comparison we observed that the CPU time of MALSAR varies quadratically with number of tasks. For example for $\alpha=0.5$ the CPU was of $3.3,4.8, \ldots$, secs. for $\mathrm{T}=20,40, \ldots, 1200$

From these results, we are able to claim that the standard MTL implementation has a quadratic complexity with respect to the number of tasks, whereas our ADMM implementation is able to reach the same optimal solution in a fixed number of outer steps.

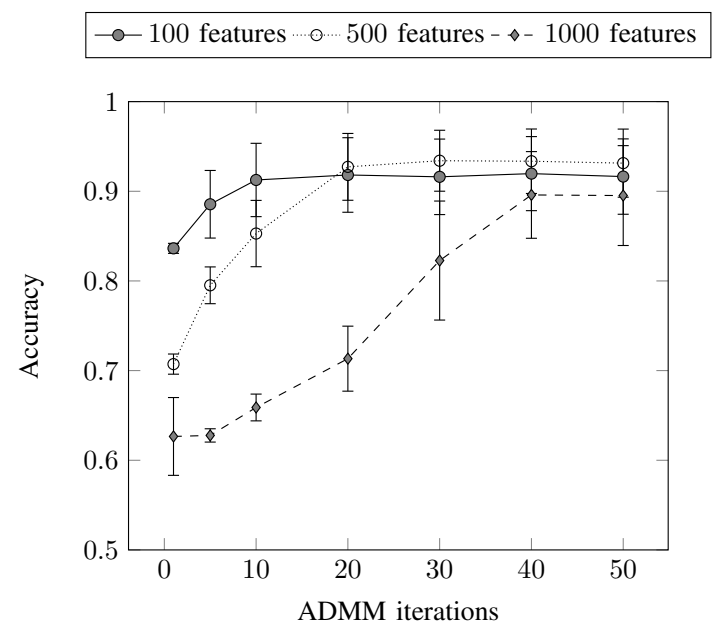

Fig. 2. Performance of ADMM with different dimensionality

\section{B. URL classification}

The next dataset was extracted from a production environment in an online advertising firm. For online targeting, a data provider supplies data to an advertiser indicating which users are interested in specific product classes. In order to determine the interest of each user, web pages are first classified into interest categories (eg 'fashion', 'photography' etc). Thereafter the browsing behavior of users can be used to predict their interests. One way of achieving it is to predict the category of a page from the contents of a url. We can tackle this task using a bag-of-words strategy [Joachims, 2002]. Modern

\begin{tabular}{|c|c|c|c|c|c|}
\hline \multirow{2}{*}{ Tasks } & MALSAR time (s) & \multicolumn{4}{|c|}{ ADMM steps } \\
\cline { 2 - 6 } & $\alpha=0.5$ & $\alpha=0.0$ & $\alpha=0.1$ & $\alpha=0.5$ & $\alpha=1.0$ \\
\hline 20 & 3.3 & 21 & 16 & 10 & 10 \\
40 & 4.8 & 21 & 16 & 11 & 11 \\
60 & 6.4 & 21 & 16 & 10 & 10 \\
80 & 8.3 & 21 & 16 & 10 & 10 \\
100 & 10.7 & 21 & 16 & 10 & 10 \\
200 & 33.1 & 21 & 16 & 11 & 11 \\
400 & 95.8 & 21 & 16 & 11 & 11 \\
800 & 342.5 & 21 & 16 & 11 & 11 \\
1200 & 698.3 & 21 & 16 & 10 & 10 \\
\hline
\end{tabular}

TABLE I

CPU TIME OF MALSAR ALGORITHM AND NUMBER OF OUTER STEPS OF OUR ADMM ALGORITHM IN ORDER TO REACH THE SAME ACCURACY OF MALSAR, WITH DIFFERENT AMOUNTS OF TASKS. THE VARIABLE $\alpha$ IS EQUAL TO $\alpha=\frac{\lambda_{2}}{\lambda_{1}+\lambda_{2}}$

urls carry enough information to identify the page contents thanks to Search Engine Optimization strategies. Before being able to apply this, words should be detected and segmented into meaningful $\mathrm{n}$-grams. This process can be easily achieved though a Viterbi-like strategy. The details of the algorithm used can be found in [Segaran et al., 2009. Ch. 4]. It calculates the most likely segmentation of a string containing text with no spaces based on a Viterbi-like probability maximization process and the prior probabilities of different n-grams extracted from the 1 Trillion words dataset gathered by Google [Brants et al., 2006]. Once the URL is segmented, a bag-ofwords representation is built.

The dataset treated is a collection of urls extracted from

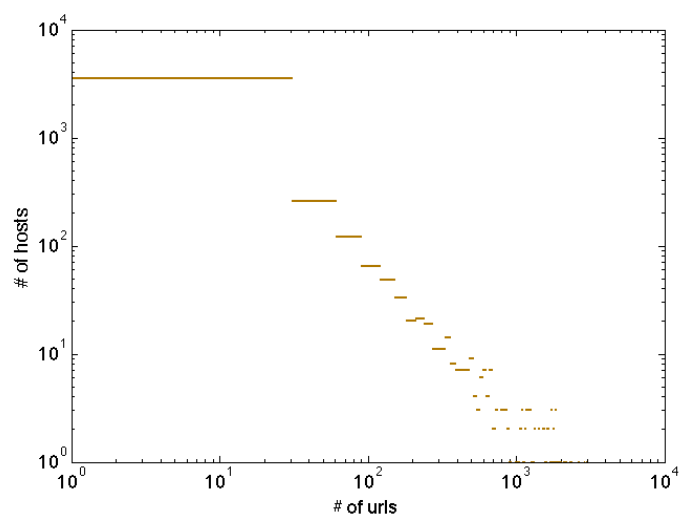

Fig. 3. \# of patterns per task (client) in the URL classification dataset.

the client base at Skimlinks labeled by humans as related to fashion or not. The dataset is a compound of over 500,000 urls extracted from 4,326 different client hosts embedded in a 150,000 dimension bag of words vector space. Thus, we have a binary classification task for a set of different tasks (clients/hosts). We hypothesize that different clients may use different vocabularies and ways of building urls, but that the underlying set of n-grams that indicate fashion should be shared between all of them. In addition, we can see in Figure 3 that the number of urls extracted from each client is highly skewed, so most tasks do not have enough data to 


\begin{tabular}{|c|c|c|c|}
\hline Method & Validation & Train & Test \\
\hline $\begin{array}{c}\text { Linear SVM } \\
\text { single task } \\
\text { Linear SVM }\end{array}$ & $0.780_{ \pm 0.014}$ & $0.813_{ \pm 0.021}$ & $0.768_{ \pm 0.083}$ \\
individual tasks & $0.793_{ \pm 0.001}$ & $0.926_{ \pm 0.027}$ & $0.811_{ \pm 0.001}$ \\
ADMM $_{5}$ & $0.801_{ \pm 0.001}$ & $0.918_{ \pm 0.007}$ & $0.814_{ \pm 0.001}$ \\
ADMM $_{30}$ & $0.817_{ \pm 0.001}$ & $0.887_{ \pm 0.003}$ & $0.836_{ \pm 0.001}$ \\
ADMM $_{50}$ & $0.817_{ \pm 0.001}$ & $0.891_{ \pm 0.003}$ & $0.835_{ \pm 0.001}$ \\
\hline
\end{tabular}

TABLE II

Classification ACCURACY $\pm s t d_{\text {FOR }}$ SKIMLINKS DATASET.

build a reliable model in such a high dimensional space. This problem's scenario is similar to the artificial case previously treated and so we expect that sharing information between tasks could be beneficial.

Table $\Pi$ summarizes the results for the categorization dataset when compared to its single task and individual tasks counterparts. As it can be observed in the third column, there is an expected gain of $6 \%$ accuracy when compared with a single SVM model trained for all the the tasks and of 3\% when compared to an SVM trained individually for each task. When comparing these results with the distribution of patterns per task, we can observe that there is enough variety between tasks that hinders SVM capacity to adapt to all of them. On the other hand, for less populated tasks there is not enough data to achieve a high accuracy and so all the information is not exploited. The capacity to transfer information embedded in MTL seems to balance the right amount of regularization and information sharing, giving the best results.

One of the questions in systems where an individualised response is needed is what to do in a cold start situation, i.e. what response to provide when we do not have data for some individual task. Single model training would advocate to use the same model as for all the tasks, whereas if we choose to have individualised models, we would lack the model for that case. It seems natural to think that the average model $\mathbf{w}_{0}$ in formula (2), which constitutes the bias for any task in the context, would be the right answer. In the previous dataset we discarded 29753 patterns from unpopulated task. So these tasks would be a sample of "cold start" of tasks we do not have data on. The accuracy of $\mathbf{w}_{0}$ and of the single task SVM previously obtained are $82 \%$ and $80 \%$ respectively. When unified with the results in Table II we can see that MTL shows a good adaptation to individual tasks and, at the same time, a good prior bias is also discovered.

\section{CONClusions And Future Work}

In this work, we presented an algorithm for distributed MTL with task variance regularization. The iterations of the proposed algorithm are completely parallel and an accurate solution can be obtained in tens of iterations. The approach decouples the training of the different tasks by making use of an ADMM optimization scheme. We have tested the approach both on an artificial and on a real large scale datasets and proven that this MTL method can scale up to real large scale problems with better accuracy. We hope that our results will encourage further research on scaling up other MTL methods and their application to real heterogeneous databases. The method presented could be readily extended to more general MTL regularizers of the form $\sum_{s, t=1}^{T}\left\langle\mathbf{w}_{s}, \mathbf{w}_{t}\right\rangle G_{s t}$. An interesting question is for which classes of positive definite matrices $G$ distributed optimization over the auxiliary variables $\mathbf{z}_{t}$ would still be possible, like in the case studied in this paper. For example, this should be possible when matrix $G$ is the graph Laplacian of a tree as in [Khosla et al., 2012]. Yet another extension of the method presented in this paper arises in the context of multitask latent subcategory models as in [Stamos et al., 2015], where our algorithm could be employed to solve large scale image classification and detection problems for computer vision. Finally, ideas from [Suzuki, 2013] may be employed to obtain fully stochastic versions of our method.

Acknowledgements: David Martinez Rego was supported by the Xunta de Galicia through the postdoctoral research grant POS-A/2013/196. We wish to thank Patrick Combettes, Taiji Suzuki and Yiming Ying for valuable comments.

\section{REFERENCES}

[Ahmed et al., 2014] A. Ahmed, A. Das, A.J. Smola. Scalable hierarchical Multitask Learning Algorithms for Conversion Optimization in Display Advertising. ACM International Conference on Web Search and Data Mining, pages 153-162, 2014.

[Bai et al., 2012] J. Bai, K. Zhou, G. Xue, H. Zha, Z. Zheng, Multi-task learning to rank for web search. Pattern Recognition Letters, 33:173-181, 2012.

[Baxter et al., 2000] J. Baxter. A model of inductive bias learning, Journal of Artificial Intelligence Research, 12:149-198, 2000.

[Ben-David et al., 2003] S. Ben-David, R. Schuller. Exploiting Task Relatedness for Multiple Task Learning, SIGKDD, pages 567-580, 2003.

[Birlutiu et al., 2013] A. Birlutiu, P. Groot, T. Heskes. Efficiently learning the preferences of people. Machine Learning, 90:1-28, 2013.

[Brants et al., 2006] T. Brants and A. Franz. Web 1T 5-gram Version 1. Philadelphia: Linguistic Data Consortium, 2006.

[Bottou et al., 2008] L. Bottou and O. Bousquet. The Tradeoffs of Large Scale Learning. NIPS, 2006.

[Boyd, 2010] S. Boyd, N. Parikh, E. Chu, B. Peleato and J. Eckstein. Distributed Optimization and Statistical Learning via the Alternating Direction Method of Multipliers. Foundations and Trend in Machine Learning, 3:1-122, 2010.

[Caruana, 1997] R. Caruana. Multi-task learning. Machine Learning, 28:4175, 1997.

[D’Avanzo et al. 2013] C. D’Avanzo, A. Goljahani, G. Pillonetto, G. De Nicolao, G. Sparacino. A multi-task learning approach for the extraction of single-trial evoked potentials. Journal Computer Methods and Programs in Biomedicine 110:125-136, 2013.

[Dinuzzo et al., 2011] F. Dinuzzo, G. Pillonetto, G. De Nicolao. Clientserver Multitask Learning from distributed datasets. IEEE Transactions on Neural Networks, 22:290-303, 2011.

[Wang et al., 2015] J. Wang, M. Kolar, N. Srebro. Distributed Multitask Learning. arXiv preprint, Oct 2015.

[Eckstein and Bertsekas, 1992] J. Eckstein and D.P. Bertsekas. On the Douglas-Rachford splitting method and the proximal point algorithm for maximal monotone operators. Mathematical Programming 55(1-3):293318, 1992.

[Evgeniou and Pontil, 2004] T. Evgeniou, M. Pontil. Regularized Multi-Task Learning. SIGKDD, 2004.

[Goldstein et al., 2014] T. Goldstein, B. O'Donoghue, S. Setzer and R. Baraniuk. Fast alternating direction optimization methods. SIAM Journal on Imaging Sciences, 7(3), 1588-1623, 2014.

[He et al, 2012] B. He and X. Yuan. On the $O(1 / n)$ Convergence Rate of the Douglas-Rachford Alternating Direction Method. SIAM J. Numer. Anal., 50(2):700-709, 2012.

[Hong, 2013] M. Hong and Z. Luo. On the Linear Convergence of the Alternating Direction Method of Multipliers, arXiv:1208.3922v3, 2013. 
[Hsieh et al., 2008] C. Hsieh, K. Chang, S. Sathiya Keerthi, S Sundararajan. A Dual Coordinate Descent Method for Large-scale SVM. ICML, 2008.

[Huang et al., 2013] S. Huang, W. Peng, J. Li, D. Lee, Sentiment and topic analysis on social media: a multi-task multi-label classification approach. Proceedings of the 5th Annual ACM Web Science Conference, pages 172-181, 2013.

[Khosla et al., 2012] A. Khosla, T. Zhou, T., Malisiewicz, A.A. Efros, A. Torralba. Undoing the damage of dataset bias. In Proc. ECCV, pages 158-171, 2012.

[Joachims, 2002] T. Joachims, Learning to Classify Text using Support Vector Machines, Dissertation, Kluwer, 2002.

[Segaran et al., 2009] T. Segaran, J. Hammerbacher. Beautiful Data: The Stories Behind Elegant Data Solutions. O'Reilly Media, 2009.

[Maurer et al., 2006] Andreas Maurer. Bounds for linear multitask learning. Journal of Machine Learning Research, 7:117-139, 2006.

[Mota et al., 2011] J.F.C. Mota, J.M.F. Xavier, P.M.Q. Aguiar, M. Püschel. A proof of convergence for the alternating direction method of multipliers applied to polyhedral-constrained functions, arXiv preprint arXiv:1112.2295, 2011.

[Rakhlin et al., 2012] A. Rakhlin, O. Shamir and K. Sridharan. Making Gradient Descent Optimal for Strongly Convex Stochastic Optimization. ICML 2012.

[Shalev-Shwartz et al., 2014] S. Shalev-Shwartz, S. Ben-David. Understanding Machine Learning. From Theory to Algorithms. Cambridge University Press. 2014.

[Shawe Taylor and Cristianini, 2004] J. Shawe Taylor and N. Cristianini. Kernel Methods for Pattern Analysis. Cambridge University Press, 2004.

[Silver and Mercer, 1996] D. L. Silver and R.E Mercer. The parallel transfer of task knowledge using dynamic learning rates based on a measure of relatedness. Connection Science, 8, p. 277?294, 1996.

[Thrun, 1997] S. Thrun and L. Pratt. Learning to Learn. Kluwer Academic Publishers, November 1997.

[Stamos et al., 2015] D. Stamos, S. Martelli, M. Nabi, A. McDonald, V. Murino, M. Pontil. Learning with Dataset Bias in Latent Subcategory Models. In Proceedings of CVPR, pages 3650-3658, 2015.

[Suzuki, 2013] T. Suzuki. Dual Averaging and Proximal Gradient Descent for Online Alternating Direction Multiplier Method. International Conference on Machine Learning, JMLR Workshop and Conference Proceedings 28(1): 392-400, 2013 